EPJ Web of Conferences 59, 03002 (2013)

DOI: $10.1051 /$ epjconf/20135903002

(C) Owned by the authors, published by EDP Sciences, 2013

\title{
Hydrodynamic modeling and simulations of shock ignition thresholds
}

\author{
M. Lafon ${ }^{a}$, X. Ribeyre, G. Schurtz and E. Le Bel \\ Centre Lasers Intenses et Applications, Université Bordeaux 1 - CEA - CNRS, \\ 33405 Talence, France
}

\begin{abstract}
The Shock Ignition (SI) scheme [1] offers to reduce the laser requirements by relaxing the implosion phase to sub-ignition velocities and later adding an intense laser spike. Depending on laser energy, target characteristics and implosion velocity, high gains are expected [2,3]. Relevant intensities for scaled targets imploded in the velocity range from 150 to $400 \mathrm{~km} / \mathrm{s}$ are defined at ignition thresholds. A range of moderate implosion velocities is specified to match safe implosions. These conditions for target design are then inferred for relevant NIF and LMJ shock-ignited targets.
\end{abstract}

\section{INTRODUCTION}

In Inertial Confinement Fusion (ICF), the thermonuclear fuel is imploded and assembled by a shaped laser pulse. This process involves high implosion velocities $(350-400 \mathrm{~km} / \mathrm{s})$ in order to both compress and heat the fuel up to trigger ignition into the hot spot. The SI scheme enables the ignition to occur while relaxing the implosion phase to sub-ignition velocities $(200-350 \mathrm{~km} / \mathrm{s})$ and launching a high pressure spike (100-300 TW) at the end of the main drive stage. This spike pulse will generate a shock that will converge through the precompressed target and set the final assembly on a non-isobaric configuration. Based upon this SI characteristic, the ignition could be achieved at lower laser energy than for conventional laser drive and high gains are expected [2, 3].

The hot spot ignition conditions at stagnation time depend on both the implosion velocity set by the main drive and the laser power dedicated to the spike generation. Benefiting from the independence of the compression and the heating phases, this study is seeking the optimal splitting of the laser energy to determine the relevant trade off between these two key parameters. Indeed, the reliable domains for both the implosion velocity and the spike power are limited by respectively hydrodynamic and parametric instabilities concerns. In this paper, the laser and energy power requirements for ignition, as well as the peak laser intensity, are derived depending on the target implosion velocity. The optimal implosion parameters for shock ignited target designs are then discussed.

\section{GAIN CURVES DEPENDING ON THE TARGET IMPLOSION VELOCITY}

In this study, the HiPER (High Power laser Energy Research facility) baseline target [4] is used. It consists of a $211-\mu$ m-thick cryogenic Deuterium-Tritium (DT) cryogenic shell filled with DT gas and a $1044-\mu$ m-outer radius. The simulations were performed in $1 \mathrm{D}$ spherical geometry using the hydrodynamic code CHIC [5].

\footnotetext{
ae-mail: mlaf@lle.rochester.edu
}

This is an Open Access article distributed under the terms of the Creative Commons Attribution License 2.0, which permits unrestricted use, distribution, and reproduction in any medium, provided the original work is properly cited. 


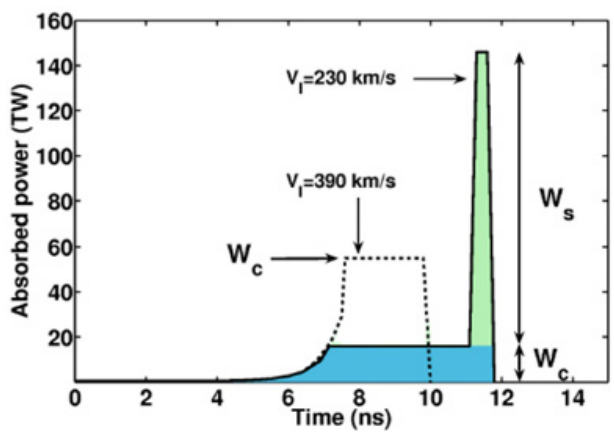

(a)

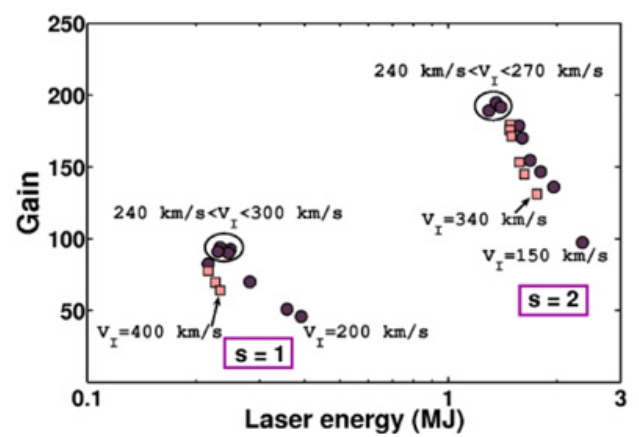

(b)

Figure 1. Laser pulses shapes desgined for ignition of the HiPER target using the conventional ignition scheme with $\mathrm{V}_{I}=390 \mathrm{~km} / \mathrm{s}$ (dashed line) and the SI scheme with $\mathrm{V}_{I}=230 \mathrm{~km} / \mathrm{s} \mathrm{(a).} \mathrm{Gain} \mathrm{curves} \mathrm{depending} \mathrm{on} \mathrm{the} \mathrm{implosion}$ velocity for the scale-1 and scale- 2 targets. Ignition is reached either by conventional scheme (square patterns) or by SI process (circle patterns) (b).

Within the framework of ICF, the target has to be imploded to high velocity at low entropy. The shell implosion is driven by the ablation pressure generated by the laser irradiation. The implosion velocity is a key parameter impacting on phenomena such as mass ablation rate, shell acceleration and growth duration of hydrodynamic instabilities. The dashed line in Fig. 1(a) shows the laser pulse shape designed to ignite the HiPER target within conventional drive. The foot of the pulse is followed by a laser rise aiming at setting the fuel on a low adiabat $(\alpha \sim 1-2)$. Then, the shell is accelerated by the ablation pressure $P_{a}$ generated by the remaining pulse shape with the compression power $W_{C}$. At the end of the pulse, the shell is coasting with the implosion velocity $V_{I}=390 \mathrm{~km} / \mathrm{s}$. To accelerate a shell of given mass to the velocity $V_{I}$, the required pressure scales as $P_{a} \sim V_{I}^{2} / A_{r 0}$ [6] where $A_{r 0}=R_{0} / \Delta R_{0}$ is the shell initial aspect ratio. Moreover, $P_{a} \sim I_{L}^{2 / 3}$ and $I_{L} \sim W_{C} / R_{0}^{2}$ where $I_{L}$ is the laser intensity. Thus, the implosion velocity set by the compression pulse scales as $V_{I} \sim W_{C}^{1 / 3} A_{r 0}^{1 / 2} / R_{0}^{2 / 3}$. The implosion velocity does not affect significantly the peak areal density of the shell $\left(\rho R \propto E_{C}^{0.33} \alpha_{i f}^{0.54} V_{I}^{0.06}\right.$ [7]) but regulates the hot spot temperature as $\left\langle T>\propto E_{C}^{0.07} \alpha_{i f}^{0.15} V_{I}^{1.25}\right.$ [7] where $E_{C}$ is the energy of the laser drive and $\alpha_{i f}$ is the shell in-flight adiabat.

Reducing the driving pressure $P_{a}$ is very useful in order to improve the stability of the shell during the implosion. However, if the main drive power $W_{C}$ is reduced, the hot spot may not be heated enough. Thus, the main drive pulse is no sufficient anymore to ignite the fuel and ignition using conventional drive fails. A solution is to add an ignitor pulse at the end of the compression pulse and then one can recover the SI scheme. The solid line in Fig. 1(b) exhibits a laser pulse imparting a velocity $\mathrm{V}_{I}=230 \mathrm{~km} / \mathrm{s}$ to the HiPER target and then compensating this sub-ignition velocity with an intense spike (with the laser power $\mathrm{W}_{S}$ ) leading to ignition.

Starting from the baseline target, homothetic-scaled target may be designed using the scaling parameter $s=R / R_{\text {ref }}$ defined as the ratio between the radius of the scaled target and the HiPER target. From the Euler equations, the shell mass $M$, the peak areal density $\rho R$, the time $t$, the laser power $W_{C}$ and laser energy $E_{C}$ respectively scale as: $M=s^{3} M_{\text {ref }}, \rho R=s \rho R_{\text {ref }}, t=s^{3} t_{\text {ref }}, W_{C}=s^{2} W_{C r e f}$ and $E_{C}=s^{3} E_{C r e f}$. The thermonuclear energy released from the combustion varies according to the remaining mass at ignition and the burn fraction $\Phi$ which itself depend on the implosion velocity through the relations $\dot{m} \propto I_{L}^{1 / 3}[6]$ and $\Phi=f(\rho R)$ with $\rho R \propto E_{L}^{1 / 3}$ [7]. Figure 1(b) shows the energy gain obtained for shell implosion velocities varying from 200 to $400 \mathrm{~km} / \mathrm{s}$ for the scale- 1 target and from 150 to $340 \mathrm{~km} / \mathrm{s}$ for the scale- 2 target. The HiPER and the scale-2 targets respectively self-ignite for implosion velocities above 340 and $265 \mathrm{~km} / \mathrm{s}$. The self-ignited targets using the conventional ignition scheme are thereby represented by square patterns while the targets ignited by the SI process are shown 


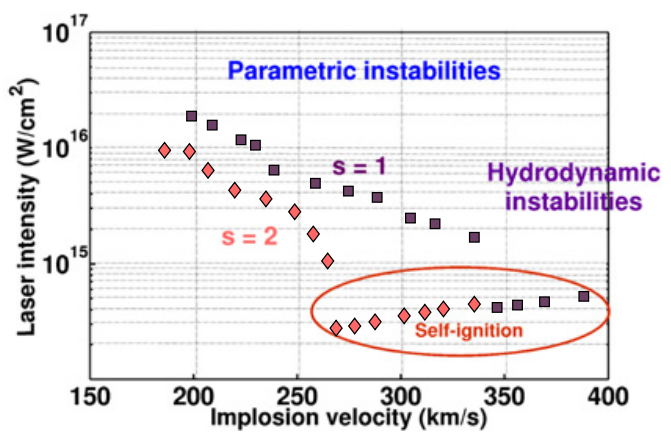

Figure 2. Peak laser intensity for ignition thresholds depending on implosion velocity for the scale-1 (square patterns) and scale-2 targets (diamond patterns).

as circle patterns. Both scale-1 and scale-2 targets point out beneficial assets of SI. For low implosion velocities, the SI process requires a very large amount of energy in the spike pulse then collapsing the energy gain while, for high implosion velocities, it is the conventional ignition scheme which needs a large amount of energy in the main drive. Nevertheless, the SI process only requires moderate amounts of energy for implosion velocities ranges from 240 to $300 \mathrm{~km} / \mathrm{s}$ for the HiPER target and 240 to $270 \mathrm{~km} / \mathrm{s}$ for the scale- 2 target, thus producing high gains. Thereby, these ranges of moderate implosion velocities turn out to be the most cost-effective ones.

\section{LASER INTENSITY FOR IGNITION THRESHOLDS}

In the SI process, the ignitor pulse needs to generate high shock pressure $(\mathrm{P} \sim 300 \mathrm{Mbar})$ which requires high intensity. The peak laser intensity is then attained when the ignitor pulse and the compression pulse overlap at the end of the laser pulse. The laser light can be absorbed in the subcritical plasma by collisional absorption if the maximum irradiance fulfils the condition $I_{L} \lambda^{2}<1$ where $I_{L}$ is in units of $10^{14} \mathrm{~W} / \mathrm{cm}^{2}$ and $\lambda$ is in $\mu \mathrm{m}$. Beyond the limit $\mathrm{I}_{L} \simeq 10^{15} \mathrm{~W} / \mathrm{cm}^{2}$ (for $\lambda=0.35 \mu \mathrm{m}$ ), the target implosion is threatened by parametric instabilities which can lead to the incident laser light scattering and may produce hot electrons in the corona then preheating the fuel and raising the adiabat [8]. The hydrodynamic instabilities may also jeopardize the shell stability, mainly by the RayleighTaylor instability growth at the external and internal shell interfaces, for implosion velocities above $\mathrm{V}_{I} \sim 350 \mathrm{~km} / \mathrm{s}[9]$.

The peak laser intensity corresponding to ignition thresholds is presented in Fig. 2 for the scale-1 (square patterns) and scale-2 (diamond patterns) targets. For implosion velocities above the respective self-ignition implosion velocity of each target, the peak intensity is slightly increasing with $\mathrm{V}_{I}$. When a spike pulse is needed, the peak intensity decreases with the rise of $\mathrm{V}_{I}$. This trend is observed for both targets. The variation of the peak intensity presents a gap between shock-ignited and self-ignited targets. At the spike launching time, the critical radius is only about half of the initial radius leading to higher peak intensities for the SI process than for the conventional ignition scheme. Indeed, for implosion velocities above the self-ignition velocity, the peak intensity is $I_{L} \sim 3-5 \times 10^{14} \mathrm{~W} / \mathrm{cm}^{2}$ while, for subignition velocities, the scale- 1 target requires $I_{L} \sim 2 \times 10^{15}-2 \times 10^{16} \mathrm{~W} / \mathrm{cm}^{2}$ to ignite and the scale-2 target needs $I_{L} \sim 1 \times 10^{15}-1 \times 10^{16} \mathrm{~W} / \mathrm{cm}^{2}$.

\section{TARGET DESIGN AND LASER REQUIREMENTS}

In this study, the numerical simulations have indicated the trade-off between the energy invested in the compression and ignition pulses in order to achieve ignition thresholds. When both components 


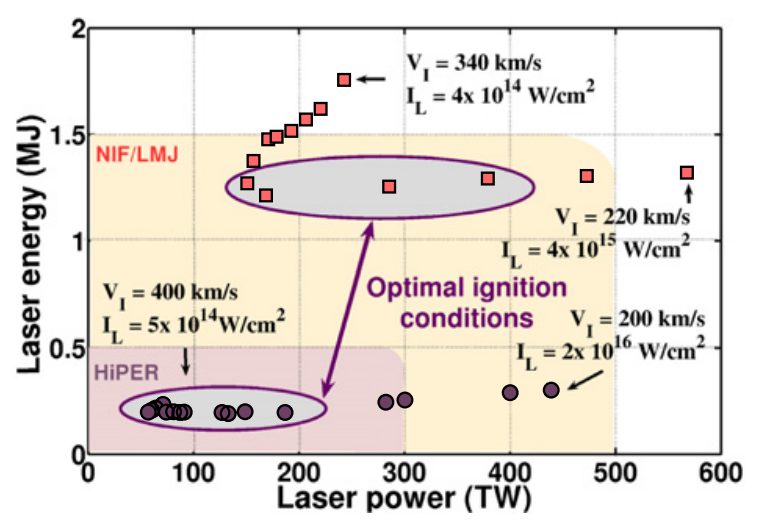

Figure 3. Incident power and energy requirements for ignition of the scale-1 (circle patterns) and scale-2 (square patterns) targets for various implosion velocities.

are considered, one can derive the total laser energy and power needed. These laser requirements are represented in a laser energy and power diagram for the HiPER target (circle patterns) and the scale- 2 target (square patterns) in Fig. 3.

These requirements for ignition thresholds evolve depending on the implosion velocity respectively from 200 to $400 \mathrm{~km} / \mathrm{s}$ and 220 to $340 \mathrm{~km} / \mathrm{s}$ for the scale- 1 and scale- 2 targets. In agreement with Sec. 2, high implosion velocities require a large amount of energy for ignition while low implosion velocities need a very high laser power. Both targets exhibit a bend in the energy-power evolution, pointing out minimal requirements in the velocity range $240 \mathrm{~km} / \mathrm{s}<V_{I}<400 \mathrm{~km} / \mathrm{s}$ for the scale-1 target and $240 \mathrm{~km} / \mathrm{s}<V_{I}<270 \mathrm{~km} / \mathrm{s}$ for the scale- 2 target. The most cost-effective numerical point designs appear to be achieved using the SI scenario. According to Sec. 2, the scale-2 target can be self-ignited with $1.3 \mathrm{MJ}$ of incident energy producing a gain $\mathrm{G} \sim 200$. Nevertheless, a reliable and more economical point design for the NIF and LMJ facilities could be described by a scale-1.2 target imploded at $\mathrm{V}_{I} \sim 250 \mathrm{~km} / \mathrm{s}$ which requires incident energy and power of about $350 \mathrm{~kJ}$ and $200 \mathrm{TW}$ and leads to a peak intensity $I_{L} \sim 4 \times 10^{15} \mathrm{~W} / \mathrm{cm}^{2}$. This point design is relevant in terms of laser requirements and safety margins for ignition on NIF and is expected to produce energy gain above 100 .

\section{CONCLUSIONS}

Scaled cryogenic DT targets have been ignited for various implosion velocities. Numerical assessments of the minimal energy of the spike pulse required for ignition are determined. The investigation of the intensity required by the ignitor shock shows that relevant targets for SI involve peak laser intensities in the range $1 \times 10^{15}-2 \times 10^{16} \mathrm{~W} / \mathrm{cm}^{2}$. For instance, the whole process of fuel assembly and hot spot heating of a DT target of $\sim 1250 \mu \mathrm{m}$ of external radius requires a laser energy of $\sim 250 \mathrm{~kJ}$ and a laser power of $\sim 50-200 \mathrm{TW}$ to produce high gain.

\section{References}

[1] R. Betti et al, Phys. Rev. Lett. 98, 155001 (2007)

[2] M. Lafon et al, Phys. Plasmas 17, 052704 (2010)

[3] L. J. Perkins et al, Phys. Rev. Lett. 103, 045004 (2009)

[4] S. Atzeni et al, Phys. Plasmas 14, 052702 (2007) 


\section{IFSA 2011}

[5] P. H. Maire et al, SIAM Journ. Sci. Comput. 29, 1781 (2007)

[6] S. Atzeni and J. Meyer-Ter-Vehn, The Physics of Inertial Fusion (Clarendon, Oxford, 2004)

[7] R. Betti and C. Zhou, Phys. of Plasma 12, 110702 (2005)

[8] O. Klimo et al, Phys. of Plasmas 18, 082709 (2011)

[9] M. Olazabal-Loumé et al, Plasma Phys. Control. Fusion 53, 015015 (2011) 